\title{
Tunable nonlocal coupling between Kondo impurities
}

\author{
D. Tutuc, ${ }^{1, *}$ B. Popescu, ${ }^{1, \dagger}$ D. Schuh, ${ }^{2}$ W. Wegscheider,${ }^{2, \ddagger}$ and R. J. Haug ${ }^{1}$ \\ ${ }^{1}$ Institut für Festkörperphysik, Leibniz Universität Hannover, Appelstrasse 2, D-30167 Hannover, Germany \\ ${ }^{2}$ Institut für Experimentelle und Angewandte Physik, Universität Regensburg, Universitätsstrasse 31, D-93053 Regensburg, Germany
}

(Received 20 May 2011; published 27 June 2011)

\begin{abstract}
We study the tuning mechanisms of Ruderman-Kittel-Kasuya-Yosida (RKKY) exchange interaction between two lateral quantum dots in the Kondo regime. At zero magnetic field we observe the expected splitting of the Kondo resonance and estimate the nonlocal coupling strength as a function of the asymmetry between the two Kondo temperatures. At finite magnetic fields a chiral coupling between the quantum dots is observed in the Kondo chessboard and we probe the presence of the exchange interaction by analyzing the Kondo temperature with magnetic field.
\end{abstract}

DOI: 10.1103/PhysRevB.83.241308

PACS number(s): 73.21.La, 73.23.Hk, 73.63.Kv

The high versatility and tunability of semiconductor quantum dots (QDs) have attracted increasing interest in the past years and their investigation as magnetic impurities has allowed even the realization of the artificial Kondo system. ${ }^{1-3}$ The Kondo effect is a signature of spin entanglement in a many-electron system, where delocalized electrons screen a localized spin, leading to the formation of a new singlet ground state. The main signature of the Kondo effect is the formation of a peak in the density of states at the impurity site due to successive spin flips at temperatures below $T_{K}$, the so-called Kondo temperature, essentially the energy scale describing the binding energy of the spin singlet formed between the localized, unpaired electron and delocalized electrons in the leads.

Kondo impurities also interact with one another via carriermediated spin-spin interactions, which compete with the local interactions that give rise to the Kondo effect. The competition between the two effects is a form of quantum entanglement between two or more spins, usually discussed in the framework of the two-impurity Kondo problem. ${ }^{4}$ The Ruderman-Kittel-Kasuya-Yosida (RKKY) is an example of such a carrier-mediated interaction. ${ }^{4}$ In QD systems, even if the observation of the RKKY-Kondo competition has been previously claimed ${ }^{5,6}$ a conclusive understanding is missing, ${ }^{7,8}$ and the observed results have also been explained in terms of a Fano antiresonance. ${ }^{9}$ While the description in terms of Fano antiresonance is suitable for high interdot hopping, the explanation in terms of RKKY is more appropriate in the limit of strongly localized electrons. In between, the two effects can coexist. ${ }^{10,11}$ Due to the fact that the RKKY interaction provides a way for spin entanglement control beyond the nearest-neighbor restraint, and its strong dependence on the Fermi wave vector, the implications to quantum information processing cannot be underestimated. ${ }^{12}$

In this Rapid Communication we study the tuning mechanisms of the RKKY exchange interaction strength in a system with two lateral QDs coupled to a central open conducting region. ${ }^{5}$ As mentioned before, $T_{K}$ describes the binding energy of the spin singlet formed between the confined electron spin and the leads, with an analytical form given by $T_{K 1(2)} \sim$ $\exp \left[-1 /\left(2 D_{C} K_{1(2)}\right)\right],{ }^{4}$ where $T_{K 1(2)}$ is the Kondo temperature of quantum dot $1(2), D_{C}$ is the density of states (DOS) in the leads, and $K_{1(2)}$ is the Kondo coupling to the leads. Assuming a continuous DOS in the central region, the RKKY exchange interaction strength is given by $J \sim K_{1} K_{2}{ }^{7}$ Therefore, by tuning the tunnel coupling between the QD and the leads, the Kondo coupling $K_{i}$ is also tuned, and with it the strength of the exchange interaction. Using this method we study how the exchange interaction strength $J$ changes by tuning the system into an asymmetric state, more explicitly, by tuning the coupling of QD1 to the central region with respect to QD2. Furthermore, a perpendicular magnetic field induces Landau levels and edge states. At high fields the edge states in the leads remain partially spin unpolarized, so the Kondo effect still can be observed in transport. ${ }^{13-17}$

Our device was produced by local anodic oxidation with an atomic force microscope (AFM) ${ }^{18-20}$ on a GaAs/Al $\mathrm{Ga}_{1-x} \mathrm{As}$ heterostructure containing a two-dimensional electron gas (2DEG), $37 \mathrm{~nm}$ below the surface (electron density $n_{e}=$ $3.95 \times 10^{15} \mathrm{~m}^{-2}$, and mobility $\mu=65 \mathrm{~m}^{2} / \mathrm{V} \mathrm{s}$ measured at 4.2 K). The structure consists of two QDs (Fig. 1), QD1 and QD2, each $\sim 150 \mathrm{~nm}$ in diameter, connected to individual drains, and to a central common reservoir, further coupled to the source reservoir $\mathrm{S}$ via a one-dimensional (1D) constriction. The distance between the QDs is $\sim 600 \mathrm{~nm}$. Using a standard lock-in technique we measure the differential conductances $g_{1}=d I_{1} / d V_{S}$ and $g_{2}=d I_{2} / d V_{S}$ across each dot in parallel by applying an ac excitation of $10 \mu \mathrm{V}$ with a frequency of $83.3 \mathrm{~Hz}$ at the source $\mathrm{S}$. The measurements were performed in $\mathrm{a}^{3} \mathrm{He} /{ }^{4} \mathrm{He}$ dilution refrigerator with an electron temperature of $\sim 80 \mathrm{mK}$. Source-drain bias dependencies revealed a charging energy of $0.25 \mathrm{meV}$ for QD1, $0.3 \mathrm{meV}$ for QD2, and single energy level spacings of $\Delta \approx 50 \mu \mathrm{eV}$. Gates G2 and G3 are used to control the coupling of the two dots to the central region, while G5 and G6 are used as plunger gates.

Both QDs can be individually tuned in the Kondo regime and, by changing the electron number one by one, we can switch from a Kondo to a non-Kondo valley. Figure 2(a) shows a Kondo resonance of QD2, or the so-called zero-bias anomaly (ZBA), as a function of dc bias, while the inset shows the corresponding situation in QD1, i.e., tuned to the middle of a Coulomb valley that does not exhibit a Kondo resonance. To estimate the Kondo temperature we use $T_{K}=(w \pi \delta) /\left(4 k_{B}\right){ }^{4}$ where $w$ is the Wilson number, $k_{B}$ is the Boltzmann constant, and $\delta$ is the half width at half maximum of the Kondo resonance. ${ }^{21}$ A value of $280 \mathrm{mK}$ is obtained for $T_{K 2}$. Changing 


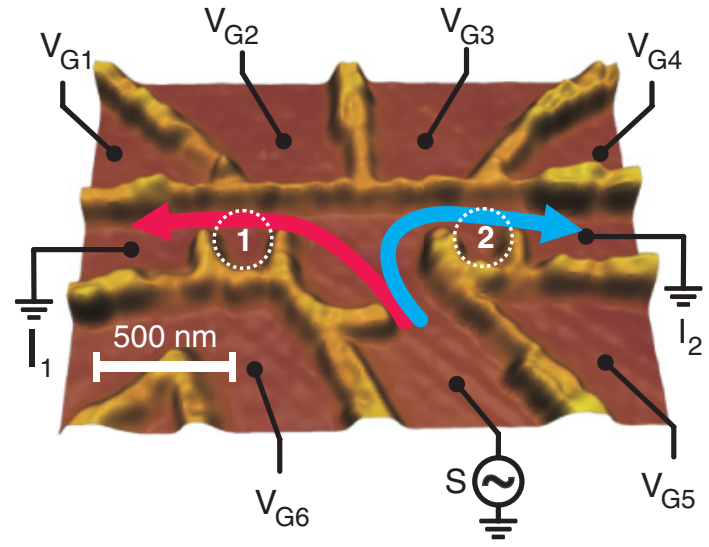

FIG. 1. (Color) AFM image of our device defined by oxide lines (bright/light brown). Quantum dots, 1 and 2, are connected to a common source $S$, and each to individual drains. Six in-plane gates, G1-G6, control the potentials of the dots and coupling to the leads. The arrows mark the measured transport paths.

the electron number in QD1 by one ( $V_{G 6}$ from -37 to $-20 \mathrm{mV}$ ) brings it into a Kondo state [inset of Fig. 2(c)], characterized by a Kondo temperature $T_{K 1} \simeq 720 \mathrm{mK}$ (Ref. 22) for $V_{G 2}=$ $-48 \mathrm{mV}$. The color plot of the differential conductance $g_{2}$ through QD2 as a function of bias voltage and gate G5 [Fig. 2(b)] shows the behavior of the QD2 ZBA when QD1 is tuned in the middle of the Kondo valley for $V_{G 2}=-54 \mathrm{mV}$. From $V_{G 5} \cong-175 \mathrm{mV}$ the ZBA starts to deviate to the right-hand side along with a decrease in conductance, which suggests the beginning of a splitting. At $V_{G 5} \simeq-167 \mathrm{mV}$ beside the Kondo peak at $\sim 75 \mu \mathrm{V}$ bias, a shoulder emerges at $\sim-70 \mu \mathrm{V}$ [marked in Fig. 2(b) by two dots and arrows]. The asymmetric peak splitting is interpreted in terms of coupling
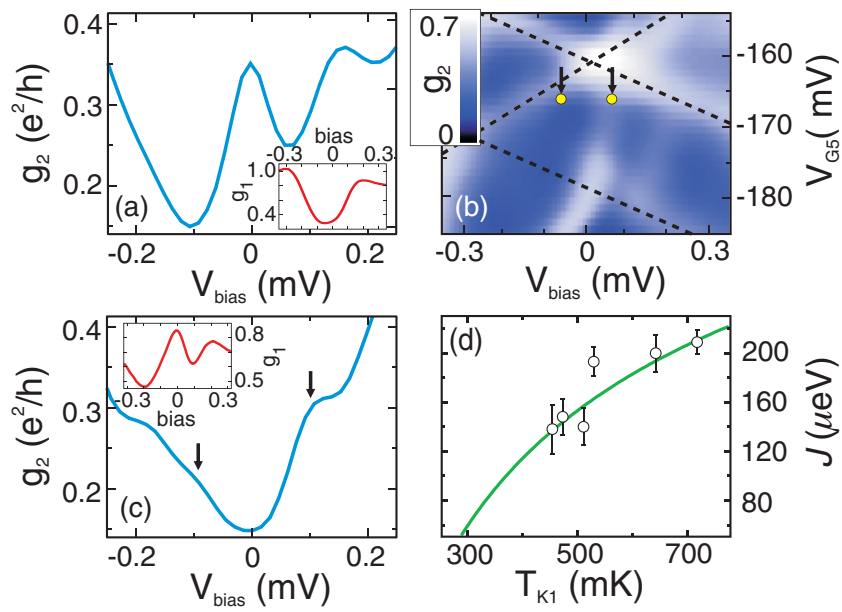

FIG. 2. (Color) (a) Conductance $g_{2}$ through QD2 showing a zero-bias anomaly, when QD1 is tuned in a non-Kondo valley (inset). (b) Color scale plot of $g_{2}$ as a function of gate G5 and bias voltage, showing the splitting of the QD2 ZBA. The dot+arrow marks the splitting. (c) Conductance $g_{2}$ showing a split zero-bias anomaly when QD1 is tuned in a Kondo state (inset) taken along the dashed line in (b). The black arrows mark the position of the split ZBA. (d) Exchange interaction strength $J$ as a function of $T_{K 1}$, fit to $1 / \ln (x)$ (line). asymmetry, QD2 being stronger coupled to the central region. The fact that we see a dependence on the Kondo energy scale indicates that the splitting is caused by the RKKY exchange interaction. The splitting of the Kondo resonance gives direct access to $J$ using the relation $e V_{\text {bias }}= \pm J / 2$ [black arrows in Fig. 2(c)] $]^{7,23,24}$ and the obtained value is $\sim 200 \mu \mathrm{eV}$. However, the ZBA splitting is observed only in QD2, while in QD1 we see just a suppression of the Kondo resonance, probably due to the strong asymmetry between the two Kondo scales. By adjusting $V_{G 2}$ between -42 and $-56 \mathrm{mV}$ the coupling of QD1 to the central region is tuned, and the Kondo temperature of QD1 at the same time. Therefore, we can measure the magnitude of the splitting as a function of $T_{K 1}$ [Fig. 2(c)]. The analytical relation for the Kondo coupling $K_{i} \sim-1 /\left[2 D_{C} \ln \left(T_{K i}\right)\right]$ gives an asymptotic behavior of the RKKY exchange interaction strength $J$ as a function of the Kondo temperature $T_{K}$, and from a fit to $J=a+b / \ln \left(T_{K 1}\right)$ [line in Fig. 2(d)], ${ }^{25}$ we obtain $a \sim 1.2 \mathrm{meV}$ and $b \sim$ $-6.5 \mathrm{meV}$, where $a$ is extracted as the maximum bandwidth of the central region, ${ }^{7}$ while $b$ contains information about the DOS in the leads.

The Kondo effect can be observed not only at zero magnetic field, but also at finite perpendicular magnetic field. Since one of its main conditions is the presence of both spin up and spin down in the leads, the observation of the Kondo effect in the magnetic field requires largely unpolarized leads. In our sample we can observe the Kondo effect up to 5.5 $\mathrm{T}$ in both QDs. This corresponds to a filling factor of the leads $v_{\text {leads }} \simeq 3$ (with $v=n_{e} h / e B$, where $n_{e}$ is the electron density, $h$ is the Planck constant, $e$ is the elementary charge, and $B$ is the magnetic field), showing that the leads are not fully polarized. In the same magnetic field range, the QD filling factors are lower, i.e., $v_{\mathrm{QD}}<v_{\text {leads }}$, and basically their properties are governed by the lowest Landau level (LLO) formed at the edge and Landau level 1 (LL1) formed at the core of the dots. Because the tunnel coupling of the core to the leads is not high enough, the Kondo effect in magnetic field involves only transport through the edge of the dot, which is closer to the leads. By changing the total spin of the edge by $\frac{1}{2}$, one can change the Kondo transport through the dot. The total spin number in the edge is given by the number of electrons, and it can be changed in two ways: Either we add an electron from the leads or from the core of the dot. The first mechanism is the result of increased voltage on a nearby plunger gate, increasing also the total electron number by one, while the second mechanism is the result of adding one flux quantum to the dot by increasing the magnetic field, and redistributing an electron from the core to the edge. ${ }^{14}$

The result of these two mechanisms is a Kondo effect modulation as a function of gate voltage and magnetic field in a regular pattern of high-low differential conductance, generally referred to as the Kondo chessboard. ${ }^{13-17}$ In Fig. 3(a) the expected Kondo chessboard pattern is shown, exhibited by QD1, with alternating regions of high (bright) and low (dark) differential conductance as a function of the voltage applied on G6 and magnetic field. In contrast, QD2 exhibits a more complicated pattern [Fig. 3(b)]. By changing the direction of the magnetic field, the situation is reversed. Now we observe a regular chessboard pattern in the transport through QD2 [Fig. 3(c)]-here the total electron number remains 

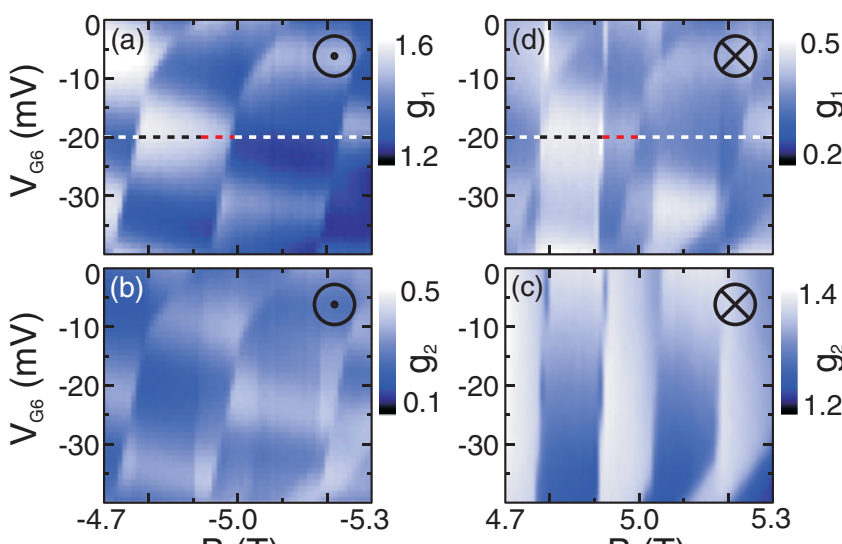

(e)
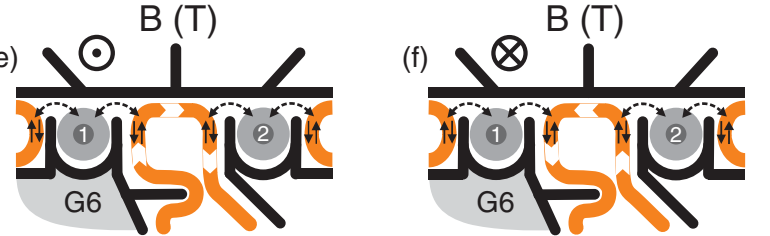

FIG. 3. (Color) Differential conductance $g_{1}$ through QD1 as a function of $V_{G 6}$ and magnetic field, for negative (a) and positive (d) magnetic field polarity. Differential conductance $g_{2}$ through QD2 as a function of $V_{G 6}$ and $B$ measured for negative (b) and positive (c) magnetic field polarity, measured simultaneously with (a) and (d), respectively. (e)-(f) Schematic diagram of the sample in magnetic field: black-AFM oxide lines; orange—edge states. Landau level 1 (dark gray) and Landau level 0 (light gray) are marked in the QDs.

unchanged, because gate G6 has a very small lever arm on QD2, and one sees only a modulation of the conductance by the magnetic field. At the same time QD1 [Fig. 3(d)] exhibits a pattern similar to the one in Fig. 3(b). The data presented in Figs. 3(a) and 3(b), respectively 3(c) and 3(d), are acquired in parallel, in the same range of $V_{G 6}$ and $B$ field, so the differences arise only from changing the polarity of the perpendicular magnetic field, which in fact changes the direction of electron transport in the edge states formed in the central region between the dots. In the first case [Figs. 3(a) and 3(b)], the edge state picture corresponds to the one depicted in Fig. 3(e), that is, the edge state transport direction is clockwise in the central region, therefore transport through QD2 will be sensitive to potential changes in the edge states generated by electron transport through QD1, i.e., the sample will behave as a current divider. As a consequence, QD2 will exhibit a combination of its own chessboard pattern and a negative of the QD1 pattern [Fig. 3(b)]. In the second situation [Figs. 3(c) and $3(\mathrm{~d})$ ] the edge state transport direction is reversed-as depicted in Fig. 3(f), i.e., the electrons move counterclockwise in the edge states in the central region. Hence, now QD1 will exhibit a combination of both chessboard patterns [Fig. 3(d)]. The measurements in Figs. 3(a)-3(d) demonstrate a chiral coupling between the QDs via the edge states formed in the central region. Using these conductance plots one can identify the regions where only one or both QDs exhibit transport through a Kondo state. Along the dashed lines in Figs. 3(a) and $3(\mathrm{~d})$ two such situations are marked, that is, between \pm 4.77 and $\pm 4.97 \mathrm{~T}$ (black and red part on the dashed line) QD1 exhibits transport through a Kondo state. In the \pm 4.9 to $\pm 5.04 \mathrm{~T}$ interval QD2 also exhibits Kondo transport [Fig. 3(c)], therefore from \pm 4.9 to $\pm 4.97 \mathrm{~T}$ (the red part on the dashed lines) both QDs are in the Kondo regime. It is worth mentioning that the sum of the differential conductances through the two dots does not change with the magnetic field direction, hence a departure from the Onsager symmetry relations ${ }^{27}$ is unlikely.

In order to probe the exchange interaction between the two QDs in a perpendicular magnetic field in detail, we investigate the temperature dependence of QD1 taken along the dashed lines in Figs. 3(a) and 3(d). In Fig. 4(a) the sharp steps in the differential conductance at -4.77 and $-4.97 \mathrm{~T}$ mark the onset of the Kondo effect in QD1, and it can be observed that at $\sim-4.9 \mathrm{~T}$ there is a change in the temperature dependence behavior, suggesting the presence of a second energy scale. For the opposite polarity [Fig. 4(b)], due to the change in the edge state chirality, an extra step in the conductance of QD1 marks the onset of the Kondo effect in QD2 at $\sim 4.9 \mathrm{~T}$. Using the relation $g(T)=g_{0}\left[T_{K}^{\prime} /\left(T^{2}+T_{K}^{\prime 2}\right)\right]^{s}$ with $T_{K}^{\prime}=T_{K} /\left(\sqrt{2^{1 / s}-1}\right)($ Ref. 3) to fit the measured temperature dependence within the \pm 4.77 to $\pm 4.97 \mathrm{~T}$ interval, we obtain a change in $T_{K 1}$ with magnetic field [Figs. 4(c) and 4(d)]. From $\pm 4.7 \mathrm{~T}$ up to $\sim \pm 4.9 \mathrm{~T}, T_{K 1}$ shows a monotonic decrease, which is followed by an increase up to $\sim \pm 4.95 \mathrm{~T}$, and then it decreases rapidly. The position where $T_{K 1}$ starts to increase coincides with the onset of the Kondo effect in QD2. The fitting procedure is done with the $s$ parameter left free and the obtained values are shown along with $T_{K 1}$ in Figs. 4(c) and $4(\mathrm{~d})$.

As mentioned before, at high perpendicular magnetic fields the Kondo effect involves transport only through Landau level 0 (LL0) energy states. Since the measurement is done at a fixed gate voltage, i.e., at a fixed energy, as the magnetic field is increased, the ground state moves lower in energy and the electron wave function is compressed, ${ }^{26}$ and thus the Kondo coupling is reduced, which explains the initial decrease of $T_{K 1}$. In this region $s$ shows a similar decrease with magnetic field, with values between 2.5 and 0.5 , which indicate a clear departure from the 0.22 value for spin $\frac{1}{2}{ }^{3}$


FIG. 4. (Color) Temperature dependence of QD1 for negative (a) and positive (b) magnetic field polarity, taken along the white dashed lines in Figs. 3(a) and 3(d), respectively. $T_{K 1}(\otimes)$ vs magnetic field for negative (c) and positive (d) polarity; the green open circles ( $\mathrm{O}$ ) are the $s$ fitting parameter. 
However, similar values have been previously reported in the Kondo chessboard. ${ }^{14}$ On the other hand, the increase and decrease of $T_{K 1}$ at $\sim \pm 4.95 \mathrm{~T}$, which is correlated with the onset of the Kondo effect in the other QD, cannot be explained by single dot physics, although in this region, $s$ has a minimum value of 0.21 for $B=-4.96 \mathrm{~T}$, respectively 0.15 for $B=4.95 \mathrm{~T}$, remarkably close to the 0.22 value. Therefore, a nonlocal exchange interaction has to be considered. The zero-bias temperature dependence of a split ZBA (either by magnetic field or by RKKY) follows that of the zero magnetic field Kondo resonance at high temperatures, ${ }^{11,28}$ and a change in the ZBA splitting will appear as a change in the Kondo temperature. Therefore, the $T_{K 1}$ peaks seen in Figs. 4(c) and 4(d) are probably not due to an actual increase in the Kondo coupling, but are attributed to a change in ZBA splitting in the presence of the exchange interaction between the spins of the dots. Even though by reversing the magnetic field direction the electron transport direction is changed, the general behavior of $T_{K 1}$ is very similar for both magnetic field directions
[Figs. 4(c) and 4(d)], questioning whether the chirality of the edge states influences also the exchange interaction between the spins of the dots.

In conclusion, we have investigated the exchange interaction between the two quantum dots as a function of Kondo temperature asymmetry and perpendicular magnetic field. At $B=0$ we find the expected $\sim 1 / \ln \left(T_{K}\right)$ dependence of the RKKY interaction strength $J$ on the Kondo energy scale. At finite magnetic fields we observe a chiral coupling between the quantum dots in the Kondo regime, and we probe the presence of the RKKY exchange interaction by a Kondo temperature analysis.

The authors would like to thank N. Ubbelohde, C. Fricke, and F. Hohls for their help with the measurement setup and data analysis, as well as E. Räsänen, J. Martinek, R. Zitko, and R. Lopez for many valuable discussions. We acknowledge financial support from the German Excellence Initiative via QUEST and the NTH School for Contacts in Nanosystems. *tutuc@nano.uni-hannover.de

${ }^{\dagger}$ Present address: School of Engineering and Science, Jacobs University Bremen, Campus Ring 1, D-28759 Bremen, Germany.

${ }^{\ddagger}$ Present address: Laboratorium für Festkörperphysik, ETH Zürich, Schafmattstrasse 16, CH-8093 Zürich, Switzerland.

${ }^{1}$ D. Goldhaber-Gordon et al., Nature (London) 391, 156 (1998).

${ }^{2}$ S. M. Cronenwett et al., Science 281, 540 (1998).

${ }^{3}$ D. Goldhaber-Gordon et al., Phys. Rev. Lett. 81, 5225 (1998).

${ }^{4}$ A. C. Hewson, The Kondo Problem to Heavy Fermions (Cambridge University Press, Cambridge, UK, 1993).

${ }^{5}$ N. J. Craig et al., Science 304, 565 (2004).

${ }^{6}$ S. Sasaki et al., Phys. Rev. B 73, 161303 (2006).

${ }^{7}$ P. Simon et al., Phys. Rev. Lett. 94, 086602 (2005).

${ }^{8}$ M. G. Vavilov and L. I. Glazman, Phys. Rev. Lett. 94, 086805 (2005).

${ }^{9}$ G. B. Martins et al., Phys. Rev. Lett. 96, 066802 (2006).

${ }^{10}$ R. Zitko, Phys. Rev. B 81, 115316 (2010).

${ }^{11}$ J. Martinek and R. Zitko (private communication).

${ }^{12}$ D. Loss and D. P. DiVincenzo, Phys. Rev. A 57, 120 (1998).

${ }^{13}$ M. Keller et al., Phys. Rev. B 64, 033302 (2001).
${ }^{14}$ C. Fühner et al., Phys. Rev. B 66, 161305 (2002).

${ }^{15}$ M. Stopa et al., Phys. Rev. Lett. 91, 046601 (2003).

${ }^{16}$ D. Kupidura et al., Phys. Rev. Lett. 96, 046802 (2006).

${ }^{17}$ M. C. Rogge et al., Phys. Rev. Lett. 97, 176801 (2006).

${ }^{18}$ M. Ishii and K. Matsumoto, Jpn. J. Appl. Phys. 34, 1329 (1995).

${ }^{19}$ R. Held et al., Appl. Phys. Lett. 73, 262 (1998).

${ }^{20}$ U. F. Keyser et al., Appl. Phys. Lett. 76, 457 (2000).

${ }^{21}$ The HWHM of the ZBA is obtained from a Lorenz fit with 0 background.

${ }^{22}$ The Kondo temperature of each QD was measured while the other dot was not in a Kondo state.

${ }^{23}$ R. Lopez, R. Aguado, and G. Platero, Phys. Rev. Lett. 89, 136802 (2002).

${ }^{24}$ R. Aguado and D. C. Langreth, Phys. Rev. Lett. 85, 1946 (2000).

${ }^{25}$ Due to limited data range, a linear fit would work as well.

${ }^{26}$ L. Onsager, Phys. Rev. 37, 405 (1931).

${ }^{27}$ V. Fock, Z. Phys. 47, 446 (1928); C. G. Darwin, Proc. Cambridge Philos. Soc. 27, 86 (1930).

${ }^{28}$ T. A. Costi, Phys. Rev. Lett. 85, 1504 (2000). 